\title{
Divergence of categorical and coordinate spatial processing assessed with ERPs
}

\author{
Rob H.J. van der Lubbe ${ }^{\mathrm{a}, \mathrm{b}, *}$, Marieke L. Schölvinck ${ }^{\mathrm{a}, \mathrm{d}}$, J. Leon Kenemans ${ }^{\mathrm{a}, \mathrm{c}}$, Albert Postma ${ }^{\mathrm{a}}$ \\ ${ }^{a}$ Department of Psychonomics, Utrecht University, Utrecht, The Netherlands \\ ${ }^{\mathrm{b}}$ Department of Psychonomics and Human Performance Technology, Universiteit Twente, Postbus 217, 7500 AE Enschede, The Netherlands \\ ${ }^{\mathrm{c}}$ Department of Psychopharmacology, Utrecht University, Utrecht, The Netherlands \\ ${ }^{\mathrm{d}}$ Department of Physiology, University College, London, United Kingdom
}

Available online 2 March 2006

\begin{abstract}
The spatial relation between two objects may be described either precisely or more coarsely in abstract terms, denoted as coordinate and categorical descriptions, respectively. These descriptions may reflect the outcomes of two spatial coding processes, which are realized in the leftand right-hemisphere. Support for this account comes from visual field effects in categorical and coordinate judgment tasks and from patient studies. In the current study, this hypothesis was tested by using event-related potentials (ERPs) and source localization. ERPs yield information about the processing stage at which the hypothesized categorical and coordinate processing diverge due to different task demands, especially in our S1-S2 version of the Bar Dot task. A centrally presented Bar Dot (S1) was followed after $2.5 \mathrm{~s}$ by a second one (S2) in the left or right visual field; participants had to judge whether S2 matched S1 at the categorical, or, in a second task, at the coordinate level. Behavioral measures revealed a left-field advantage in the coordinate task that was absent in the categorical task. S1s elicited stronger early and late bilateral posterior responses in the coordinate than in the categorical task, possibly related to a compensatory strategy at the level of encoding and spatial memory. S2s elicited only stronger early contralateral responses, and stronger late right-hemisphere responses in the categorical task. It is proposed that the left-field advantage in the coordinate task may be due to differences in spatial resolution in perceptual encoding of the left- and right-hemispheres that are largely unaffected by the task at hand.
\end{abstract}

(C) 2006 Elsevier Ltd. All rights reserved.

Keywords: Spatial processing; Categorical; Coordinate; Visual half field; ERPs; Dipole sources

\section{Introduction}

According to Kosslyn and coworkers, the spatial relation between two objects can be described in two qualitatively different ways; either in broad categorical terms or on the basis of precise coordinates (Kosslyn, 1987; Kosslyn et al., 1989; Kosslyn, Thompson, Gitelman, \& Alpert, 1998). The first way refers to an abstract description of the relation between objects (e.g. when using words such as above or below), which can be related to theories on object identification using structural descriptions to specify spatial relations among parts (Biederman, 1987). These descriptions seem especially useful for viewpoint independent object recognition (e.g. recognizing that an object is a chair on the basis of the relations among its compounding

\footnotetext{
* Corresponding author. Tel.: +31 534893585 .

E-mail address: R.H.J.vanderLubbe@gw.utwente.nl (R.H.J. van der Lubbe).
}

parts), but may also be important for processing and memorizing the location of objects. The second way refers to a description of the precise spatial relation (i.e. the distance) between two objects, which seems essential for motor acts such as the accurate reaching towards objects. For example, to pick up one's cup of coffee, the approximate distance between one's hand and the cup needs to be estimated. In principle, these two types of descriptions could be different outcomes of a single underlying spatial coding process, but they may also reflect the outcomes of two separate types of spatial processing. The latter possibility was first proposed by Kosslyn et al. (1989), which will be denoted as the separate spatial coding hypothesis.

A critical aspect of the separate spatial coding hypothesis is the idea that distinct neural circuits are involved in computing these different descriptions. On the basis of computational modeling, Kosslyn, Chabris, Marsolek, and Koenig (1992) argued that when two tasks rely on distinct computations, a split network performs better because of reduced interference between the different computations. Combining this argument for separate 


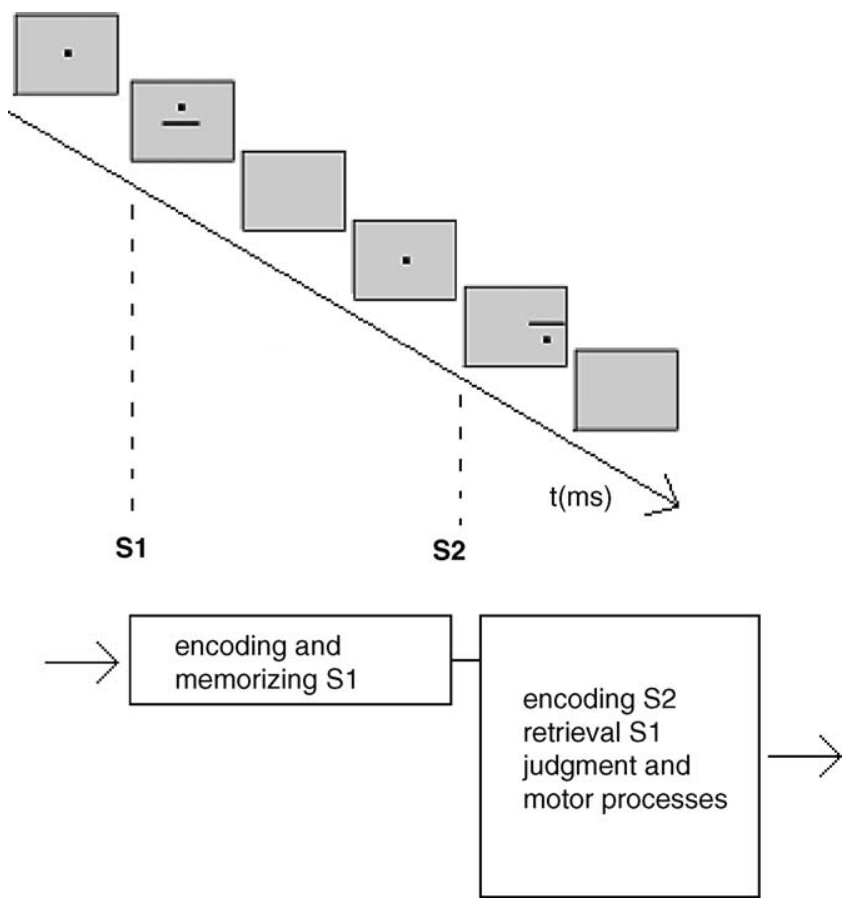

Fig. 1. An example of a trial. S1 was presented for $150 \mathrm{~ms}$ in the center of the visual field (CVF). After a blank interval of $2000 \mathrm{~ms}$, the fixation point reappeared, and $500 \mathrm{~ms}$ later S2 appeared in the left or right visual field (LVF or RVF). In the categorical task, participants had to indicate whether the position of the dot relative to the bar (above or below) was the same or different for S1 and $\mathrm{S} 2$, and in the coordinate task they had to indicate whether the distance between the dot and the bar was the same or different for S1 and S2. In the lower part of the figure, we indicated that encoding and memorizing of S1 is separated in time from judgment and motor processes and from encoding of $\mathrm{S} 2$ and retrieval of S1.

neural circuits with the traditional distinction between the lefthemisphere, associated with language, which implies the facility to form abstract categorical descriptions, and the right cerebral hemisphere, associated with spatial attention and search (e.g. see Mangun et al., 1994), it may indeed be hypothesized that categorical and coordinate spatial processing are realized in the left- and right-hemisphere, respectively. Empirical results from visual half field studies with healthy subjects (Kosslyn et al., 1989), and studies with patients suffering unilateral brain damage (Laeng, 1994) seem to support this hypothesis.

In visual half field studies, to be judged stimuli are presented briefly in the left or the right visual field (LVF or RVF), which should yield initial and more extensive processing in the contralateral hemisphere. The idea behind this manipulation, introduced by Kosslyn et al. (1989), is that for LVF stimuli the contralateral right-hemisphere is the one that is specialized for coordinate processing, whereas for RVF stimuli the contralateral left-hemisphere is specialized for categorical processing. Therefore, coordinate judgment should be faster and more accurate for LVF than for RVF stimuli, while the reverse holds for categorical judgments. Most visual half field studies employed versions of the Bar Dot task originally designed by Hellige and Michimata (1989), in which a dot appears at various distances either above or below a bar (see Fig. 1). An above/below judgment of the dot relative to the bar is supposed to require categorical process- ing, while coordinate processing is thought to be involved when judging the distance, near or far (relative to an earlier reference stimulus), between the dot and the bar. Several studies (Banich \& Federmeier, 1999; Cowin, Roth, \& Hellige, 1994; Kosslyn et al., 1989, 1998; Laeng \& Peters, 1995; Parrot, Doyon, \& Cardebat, 1998; Parrot, Doyon, Demonet, \& Cardebat, 1999; Wilkinson \& Donnelly, 1999) found support for the dependency of the visual field effect on the required type of judgment, but there were some exceptions (Bruyer, Scailquin, \& Coiboin, 1997; Sergent, 1991a, 1991b; see also Jager \& Postma, 2003). In addition, a number of studies (Cowin et al., 1994; Kosslyn et al., 1989; Michimata, 1997; Rybash \& Hoyer, 1992) revealed that practice effects may introduce a confounding as the LVF advantage in case of coordinate judgments disappeared over time, which may be explained in terms of the development of new categorical spatial representations (Baciu et al., 1999; Jager \& Postma, 2003). Finally, although several studies demonstrated a LVF advantage for coordinate judgments, the reversal (a RVF advantage) for categorical judgments was either absent (Cowin et al., 1994; Hellige \& Michimata, 1989; Hellige \& Cumberland, 2001; Michimata, 1997; Rybash \& Hoyer, 1992; Wilkinson \& Donnelly, 1999) or only present in a subset of the reported experiments (Kosslyn et al., 1989; Laeng \& Peters, 1995).

Interestingly, the distinction between categorical and coordinate spatial relations has not only been applied to visual perception but also to spatial memory (Laeng \& Peters, 1995; Postma, Izendoorn, \& De Haan, 1998), mental imagery (Trojano et al., 2002), object recognition and identification (Laeng, Shah, \& Kosslyn, 1999), and spatial communication (Kemmerer \& Tranel, 2000). In all these domains the same separate spatial processes may be differentially engaged, which emphasizes the importance of the question at what particular stage of information processing differences in computing categorical and coordinate spatial relations actually originate. Are these differences already present at the level of perceptual encoding, as originally proposed by Kosslyn, or do they specifically emerge during later stages of information processing involved with maintenance in and retrieval from memory, or even with response decision making? In all the aforementioned domains these possibilities are more or less applicable. Thus, the principal goal of the current study was to assess the level at which categorical and coordinate spatial processing start to diverge due to different task instructions.

As mentioned above, empirical results from visual half field studies with the Bar Dot task do not unambiguously support the separate spatial coding hypothesis. One problem with this technique is that half field stimuli are eventually also processed in the ipsilateral hemisphere, which may conceal differences in the involvement of the two hemispheres. Another reason may be that hemispheric specialization for categorical and coordinate processing is relative rather than absolute (Sergent, 1991a, 1991b) and that the degree of laterality differs between individuals. Specifically, both hemispheres may be involved in categorical and coordinate computations, but the left- hemisphere is more specialized in the former, whereas the right-hemisphere is more specialized in the latter type of computations. Indeed, this possibility has repeatedly been raised by Kosslyn et al. (1992). 
A more direct and sensitive test of the separate spatial coding hypothesis may be performed by using neuroimaging measures. For example, on the basis of event-related potentials (ERPs) derived from the electroencephalogram, or functional magnetic resonance imaging (fMRI) and positron emission tomography (PET), it can be assessed whether the right (or left) hemisphere is more involved in a coordinate task than in a categorical task (or vice versa).

Parrot et al. (1998) determined ERPs during the original Bar Dot task, and observed differences in activity between categorical and coordinate judgments from 230 to $270 \mathrm{~ms}$ after stimulus onset, with larger negativity above right occipito-parietal areas in the coordinate condition. This finding may indicate that categorical and coordinate spatial processing diverge at the level of perceptual encoding. However, no specific involvement above left brain areas was reported in case of categorical judgments. Result of PET and fMRI studies (Baciu et al., 1999; Kosslyn et al., 1998; Trojano et al., 2002) with comparable tasks provided more support for the involvement of the left-hemisphere. Specifically, in their PET study, Kosslyn et al. (1998) found that the left-hemisphere was generally more active during categorical judgment tasks whereas the right-hemisphere was more involved during coordinate tasks. In an fMRI study, Baciu et al. (1999) compared activation in the left and right angular gyri and found stronger activation of the left angular gyrus in the categorical task, and stronger activation of the right angular gyrus in the coordinate task. The latter data seem to support the separate spatial encoding hypothesis, although they do not allow the inference that both task differences are located at the level of stimulus encoding as the hemispherical differences may also involve a later processing stage.

In the current study, we measured EEG in an S1-S2 variant of the Bar Dot task, in which a to be remembered Bar Dot (S1) had to be compared with a second Bar Dot (S2) presented in the LVF or the RVF, either on the basis of categorical or coordinate instructions. In the categorical task, participants had to compare the relative position (same or different) of the dot in S1 (i.e. above or below the bar) with the position of the dot in S2, whereas in the coordinate task, participants had to compare the exact distance (same or different) between the dot and the bar in S1 and S2. In addition to the possibility to directly assess hemispheric differences, using this variant of the Bar Dot task in combination with ERPs has several advantages. First, it enables the separation between task effects on initial encoding processes, followed by higher perceptual and memory-related processes during the S1-S2 interval, reflected in early posterior and later posterior but also more anterior ERP components. Moreover, it excludes the confounding of subsequent retrieval, judgment and motor processes as they will be carried out after S2. Secondly, ERPs yield precise information about the timing of specific effects, and source localization (Scherg, 1990) gives a rough estimate of the cortical areas that are involved in these effects. In addition, given the S1-S2 intervals of $2.5 \mathrm{~s}$, slow waves possibly related to spatial memory can be investigated. For example, Ruchkin, Johnson, Grafman, Canoune, and Ritter (1997) found right-parietal activation for spatial memory tasks, which are areas known to play a crucial role in the allocation of spatial attention (see also Awh, Jonides, \& Reuter-Lorenz, 1998; Jha, 2002). In addition, slow negative waves over left frontal brain areas are thought to reflect the retention and/or rehearsal of verbal material (Ruchkin, Johnson, Grafman, Canoune, \& Ritter, 1992; but see Bosch, Mecklinger, \& Friederici, 2001). Apart from these advantages, the current paradigm preserves the possibility to establish visual field effects on behavior, and analyses on ERPs evoked by $\mathrm{S} 2$ enables verification of the assumption that LVF and RVF stimuli initially arrive in the contralateral hemisphere. A final advantage of the S1-S2 paradigm is a reduction of the practice effect in the coordinate task, as judgments always depend on the relation between $\mathrm{S} 1$ and $\mathrm{S} 2$.

\section{Methods}

\subsection{Participants}

Eighteen male volunteers (mean age $21 \pm 2.3$ years, with normal or corrected to normal vision) participated in this experiment, who were paid approximately $€ 40$ each. The average score on the Dutch version of the revised Annett Handedness Inventory (Annett, 1970; Briggs \& Nebes, 1975) was $21.3 \pm 2.3$, with a lowest score of 16 , which implies that all participants were strongly righthanded. All participants signed a written informed consent, and the study was approved by the ethics committee of the faculty of social sciences.

\subsection{Stimuli}

The employed stimuli (see Fig. 1) were nearly identical to the stimuli used by Kosslyn et al. (1989; Exp.3). They were composed of a bar (length $\times$ width, $\left.1.38^{\circ} \times 0.02^{\circ}\right)$ and a dot $\left(0.02^{\circ}\right)$. The dot was positioned at $0.8^{\circ}, 1.6^{\circ}, 2.4^{\circ}$, $3.2^{\circ}$ or $4.0^{\circ}$ above or below the bar. The distance between the participant and the screen was $1.1 \mathrm{~m}$. The dot and bar was presented either in the center of the visual field (CVF; S1), or in either the LVF or the RVF (S2), with the bar centered at $3.38^{\circ}$ to the left or right from a fixation point $\left(0.02^{\circ}\right)$. The fixation point, bar and dot were dark gray against a light gray background. Their luminance was set at $30 \mathrm{~cd} / \mathrm{m}^{2}$ and background luminance was set at $90 \mathrm{~cd} / \mathrm{m}^{2}$.

\subsection{Procedure}

\subsubsection{General procedure}

All participants performed a categorical and a coordinate judgment task, the order of which was counterbalanced. They received oral instructions, followed by a brief summary on the screen. Six practice trials were given at the start in both tasks. On each trial S1 was followed after a delay by S2, which required a response. Responding had to be carried out bimanually, by pressing two keys simultaneously with the two index or two middle fingers, to avoid over-engagement of one hemisphere (for a comparable procedure, see Hellige \& Michimata, 1989). In the categorical task, participants had to compare the relative position of the dot in S1 (i.e. above or below the bar) with the position of the dot in S2. By pressing buttons with the index fingers they indicated that the relative positions were the same, and by pressing buttons with the middle fingers that they were different. In the coordinate task, participants had to compare the exact distance, irrespective of the categorical level (above or below), between the dot and the bar in S1 and S2. Again, by pressing buttons with the index fingers they indicated that the distances were the same, and by pressing buttons with the middle fingers that they were different. The total duration of the experiment was approximately $3 \mathrm{~h}$. Both tasks consisted of twelve 6 min blocks, with a $1 \mathrm{~min}$ pause in between those blocks.

\subsubsection{Set up of a trial}

Each trial commenced with a blank screen for $750 \mathrm{~ms}$, followed by the fixation point for $500 \mathrm{~ms}$. Next, S1 was presented for $150 \mathrm{~ms}$ in the CVF, and after a blank screen for $2000 \mathrm{~ms}$, the fixation point was presented again for $500 \mathrm{~ms}$. Then $\mathrm{S} 2$ was displayed for $150 \mathrm{~ms}$ equally probable in the LVF or RVF. 
After S2, a blank screen was displayed for $2000 \mathrm{~ms}$, during which the participant was required to respond. The next trial started $1000 \mathrm{~ms}$ after the response or $500 \mathrm{~ms}$ after the end of the trial, which implies a maximal trial duration of $6550 \mathrm{~ms}$.

\subsubsection{Balancing of stimuli}

In each task, the number of S1 and S2 combinations was manipulated in order to keep the ratio 'same'/'different' responses balanced. All stimulus combinations were presented at least twice, in order to yield a sufficient amount of data for the EEG. This resulted in 600 trials per task. In both tasks, the dot in S1 occurred equally often above and below the bar, whereas the dot in S2 occurred equally often above and below the bar in the categorical task, and nearly equally often above and below the bar in the coordinate task

\subsection{Data recording and analysis}

Participants' responses on a response box with four buttons, placed on their lap, were measured by Vision Recorder (Version 1.0b). EEG was recorded continuously from 60 channels, using $\mathrm{Ag} / \mathrm{AgCl}$ ring electrodes attached to an electrocap. The electrodes were online referenced to $\mathrm{Cz}$, but were off-line referenced to the average across all EEG electrodes. The vertical and horizontal electro-oculogram (vEOG and hEOG) were measured from electrodes above and below the left eye and from the outer canthi of both eyes, respectively. Electrode resistance was kept below $5 \mathrm{k} \Omega$. Signals were passed through a BrainAmp amplifier (Brain Products $\mathrm{GmbH}$ ) and were recorded on-line at a sample rate of $250 \mathrm{~Hz}$. Measured activity was digitally filtered on line $(\mathrm{TC}=5.0 \mathrm{~s}$, low-pass $100 \mathrm{~Hz}$ ) by Vision Recorder installed on a Pentium III computer. Presentation@software (Version .046), installed on a Pentium II computer, controlled stimulus presentation on a $17 \mathrm{in}$. monitor and sent digital codes to Vision Recorder to indicate the moment and the type of stimulus. For the statistical analyses, Greenhouse-Geisser correction of the $p$-value was applied whenever appropriate.

\subsubsection{Behavioral data}

Reaction time (RT) was defined as the interval between the onset of S2 and the first key press. Responses slower than $4000 \mathrm{~ms}$ after S2, marked as slow, and responses occurring within $150 \mathrm{~ms}$ after onset of S2, termed premature responses, were excluded from RT analysis. Mean RTs and proportion of errors (PEs) per participant were statistically evaluated by analysis of variance (ANOVA) with factors task (categorical versus coordinate) and visual field (LVF versus RVF). Analyses were additionally performed with the factor block (1-6; trial 1-100, 101-200, etc.), to examine practice effects, which could affect performance in the coordinate task.

\subsubsection{EEG data}

EEG was analyzed using Vision Analyzer (Version 1.03). Time intervals after presenting S1 and S2 were analyzed separately for each condition. The mean amplitude from -100 to $0 \mathrm{~ms}$ before either $\mathrm{S} 1$ or $\mathrm{S} 2$ served as a baseline. For the S1-S2 interval, we examined activity from 0 to $2000 \mathrm{~ms}$ after S1, and included only those trials that were followed by correct judgments. For S2, the interval from 0 to $600 \mathrm{~ms}$ was taken for trials with correct responses. For each time interval and condition, ERPs were computed for all electrodes by averaging EEGs for all correct trials without artifacts. Lowest allowed activity was $0.10 \mu \mathrm{V}$ for $50 \mathrm{~ms}$, minimum/maximum allowed amplitude was $\pm 200,175$ and $150 \mu \mathrm{V}$, for frontal, central and parietal electrodes, respectively. Different criteria were used to avoid the exclusion of EEG data due to EOG artifacts, which induce larger amplitudes at frontal than at parietal sites. In addition, EEG was corrected for ocular artifacts by employing the method of Gratton, Coles, and Donchin (1983). Three analyses were performed. First, we examined whether early processing of $\mathrm{S} 1$ was dependent on the task instruction, by considering the $0-800 \mathrm{~ms}$ interval, with time windows of $40 \mathrm{~ms}$. To correct for the number of performed tests (Bonferroni correction), results of analyses with $p$-values less than of $0.05 / 20=0.0025$ were considered as significant. Analyses were performed on average activity in these time windows by subjecting them to an ANOVA with factors task (2), hemisphere (2), and electrode (25; i.e. for the left-hemisphere: Fp1, AF7, AF3, F7, F5, F3, F1, FT7, FC5, FC3, FC1, T7, C5, C3, C1, CP5, CP3, CP1, P7, P5, P3, P1, PO7, PO3, O1). Secondly, we examined slow wave activity within the $\mathrm{S} 1-\mathrm{S} 2$ interval, determined as the average activity from $250 \mathrm{~ms}$ time windows from 1000 to $2000 \mathrm{~ms}$ after S1 (i.e., late enough to examine memory-induced effects), which was evaluated with the same factors (a $p$-value of $0.05 / 4=0.0125$ was considered as the significance criterion). Finally, we focused on activity from 0 to $600 \mathrm{~ms}$ after S2 (to reduce the contribution of motor-related effects), by examining average activity within $40 \mathrm{~ms}$ time windows, which we evaluated with the factors task, hemisphere, electrode (25), and the additional factor visual field (LVF or RVF; a $p$-value of $0.05 / 15=0.003$ was considered as the significance criterion). For these three analyses we reported only interactive effects including the factor task, as other results are not relevant for the questions at hand, except for the possible interactions between visual field, electrode and hemisphere after S2, as they may confirm the assumption made by the visual half field technique (i.e. initial arrival in the contralateral hemisphere).

In order to specify differences in recruited brain areas between the two tasks we additionally performed source localization analyses by employing the brain electricity source analysis (BESA) algorithm (Version 2.2) on grand average ERPs per task on the moment at which the global field power reached its maximum within the relevant time intervals. BESA estimates the location and orientation of multiple dipolar sources by calculating the scalp distribution that would be obtained for a given dipole model and comparing it to the original distribution. Iterative changes in the location and orientation parameters of the sources lead to minimization of the residual variance between the model and the data. The energy criterion was set at $20 \%$ to reduce the interaction among dipoles, and the number of dipole pairs (with mirrored location and orientation parameters) was minimized to one to reduce the number of free parameters to be estimated.

\section{Results}

\subsection{Behavioral results}

Mean RTs and PEs as a function of task and visual field are shown in Fig. 2. RTs were not significantly different between the tasks ( 822 and $838 \mathrm{~ms}$ in the categorical and the coordinate tasks, respectively), $F(1,17)=0.3$, but more errors were made in the coordinate $(16.5 \%)$ than in the categorical judgment task $(6.2 \%), F(1,17)=49.1, p<0.001$. Responses tended to be faster, $F(1,17)=4.0, p=0.063$, and were more accurate, $F(1,17)=6.1$, $p=0.024$, when S2 occurred in the LVF (825 ms, 10.9\%) than when it occurred in the RVF (835 ms, 11.8\%).

A task $\times$ field interaction showed up both in RTs, $F(1,17)=4.8, p=0.043$, and PEs, $F(1,17)=5.9, p=0.026$. These findings suggest that the decrease of RT when $\mathrm{S} 2$ occurred in the LVF as compared to the RVF was present in the coordinate task (17.4 ms) but absent in the categorical task $(2 \mathrm{~ms})$, which was confirmed by separate two-sided $t$-tests (in the coordinate task, $t(17)=2.6, p=0.02$, in the categorical task, $t(17)=0.4, p=0.70)$. The effect on PEs was comparable, with increased accuracy for targets in the LVF as compared to the RVF in the coordinate task $(2.0 \%)$, and no effect in the categorical task $(-0.1 \%)$, which was also confirmed by separate $t$-tests (in the coordinate task, $t(17)=3.0, p=0.008$, in the categorical task, $t(17)=0.2$, $p=0.84)$.

Analyses with the additional factor block on RT to investigate practice effects revealed an interaction between task, field, and block, $F(5,85)=2.9, \varepsilon=0.68, p=0.036$. However, separate analyses per task revealed no effects including the factor block in the categorical task, $F \mathrm{~s}<1.4$, whereas for the coordinate task only a main effect of block was found, $F(5,85)=4.7, \varepsilon=0.45$, $p=0.013$, reflecting faster responses due to more practice. 


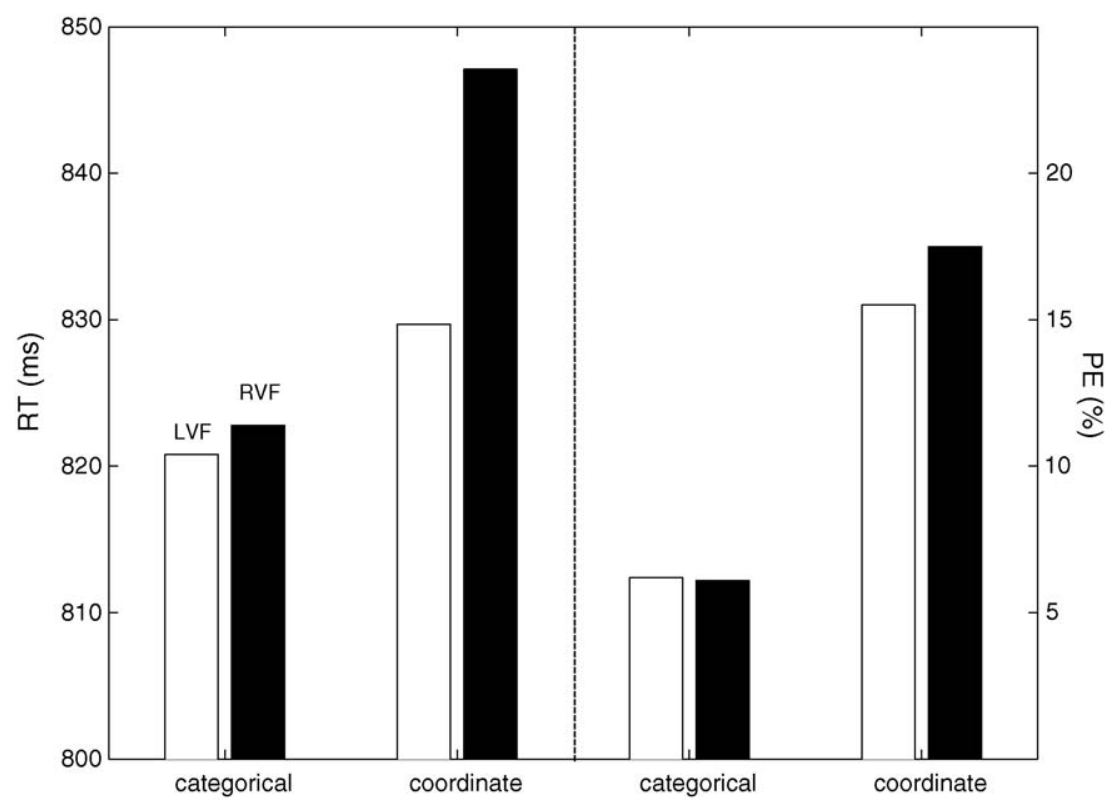

Fig. 2. RTs (left panel) and PEs (right panel) for the categorical and the coordinate tasks as a function of the visual field (LVF or RVF) in which S2 was displayed.

For PEs, we obtained an interaction between field and block, $F(5,85)=4.4, \varepsilon=0.60, p=0.008$, which reflected a decrease of accuracy $(-3.3 \% ; F(5,85)=3.3, \varepsilon=0.71, p=0.021)$ in the RVF over time, and a nonsignificant opposite trend for the LVF $(2.5 \% ; F(5,85)=2.0, \varepsilon=0.58, p=0.12)$. The latter effect was independent from the type of task, $F(5.85)=1.0$.

\subsection{ERPs and source analyses}

The percentage of trials without artifacts relative to the total number of correctly responded trials amounted to $86.4 \%$ for analyses regarding the S1-S2 intervals, and to $93.9 \%$ for analyses regarding the interval from -100 to $600 \mathrm{~ms}$ after S2.

\subsubsection{ERPs and relevant sources from 0 to $800 \mathrm{~ms}$ after $\mathrm{S} 1$}

ERPs evoked by $\mathrm{S} 1$ for relevant electrodes are displayed in Fig. 3. Task $\times$ electrode interactions were found from 160 until $320 \mathrm{~ms}, F(24,408)>6.5, p<0.002$. This effect reflected an anterior positivity, and a posterior negativity, that were more pronounced in the coordinate than in the categorical task (see Figs. 3-5). Regarding possible task dependent hemispherical differences, statistical results provided no support for this hypothesis within these time windows (task $\times$ hemisphere, task $\times$ electrode $\times$ hemisphere; $p>0.18$ ). For the other time windows, task $\times$ hemisphere interactions, $F(1,17)<4.4, \quad p>0.052$, and task $\times$ electrode $\times$ hemisphere were also not significant, $F(24,408)<1.7, p>0.18$.

The global field power of the task difference peaked at $200 \mathrm{~ms}$ after $\mathrm{S} 1$, indicating that the difference in activity between tasks was largest at this moment. Source analyses per task with one dipole pair at this time point (see Fig. 4B) left less than $4.3 \%$ residual variance, and revealed larger activity in both hemispheres for the coordinate task. In the categorical task, dipole moments for left and right-hemispheric sources, which give an indication of the strength of the sources, amounted to 2.3 and 1.7 , respectively, and in the coordinate task, dipole moments for left and right sources amounted to 3.0 and 2.5.

\subsubsection{Slow waves from 1000 to $2000 \mathrm{~ms}$ after $\mathrm{S} 1$}

Slow waves for some representative electrodes are displayed in Fig. 5. Interactions between task and electrode were present from 1000 to $2000 \mathrm{~ms}, F(24,408)>5.3, p<0.003$, which points to increased negativity in the coordinate task at posterior sites relative to the categorical task ${ }^{1}$ (see Fig. 5). The task difference was largest at about $1064 \mathrm{~ms}$ after S1. Source analyses on activity at that moment in both tasks with one dipole pair indeed revealed sources in posterior areas (see Fig. 6). Dipole moments for the left and right sources in the categorical task amounted to 0.6 and 0.3 , and in the coordinate task they amounted to 1.0 and 0.8 . Despite some small visible differences between both hemispheres, no significant task $\times$ hemisphere, and task $\times$ electrode $\times$ hemisphere interactions were found $(F \mathrm{~s}<1.1, p \mathrm{~s}>0.32)$.

\subsubsection{ERPs and relevant sources from 0 to $600 \mathrm{~ms}$ after $\mathrm{S} 2$}

ERPs evoked by $\mathrm{S} 2$ for relevant electrodes are displayed in Fig. 7. Relevant results of the statistical analyses including the factors task, electrode, hemisphere, and visual field are listed in Table 1.

The early interaction between electrode, hemisphere, and visual field, from 120 to $240 \mathrm{~ms}$, presumably reflects the arrival of $\mathrm{S} 2$ in the contralateral occipital hemisphere. Source analyses, constrained to one dipole pair at $168 \mathrm{~ms}$ revealed an extrastriate

\footnotetext{
${ }^{1}$ It should be noted that for all intervals concerning the slow waves, a main effect of electrode was found, $F(24,408)>7.4, p<0.002$, suggesting that activity was more negative at posterior sites in both tasks, which was confirmed by the source analyses.
} 

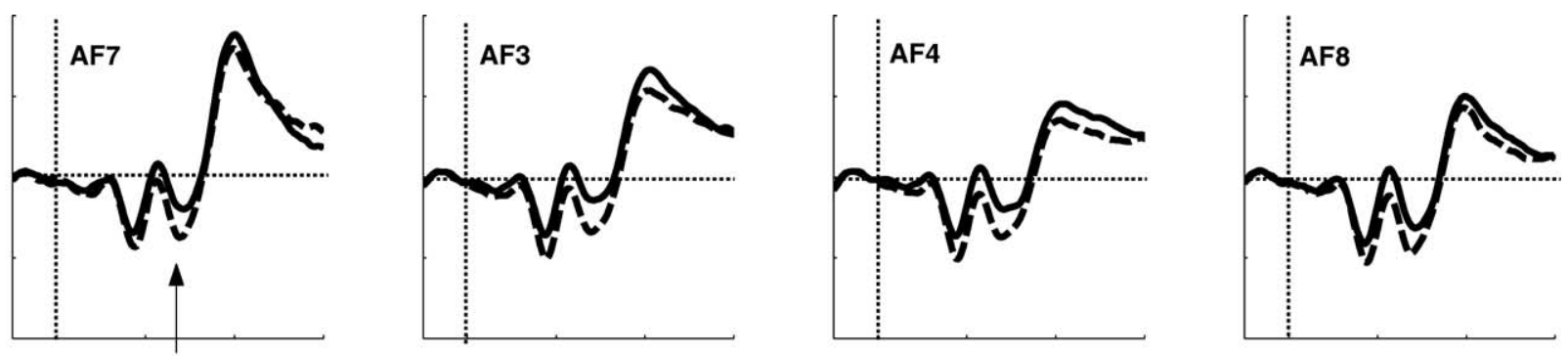

anterior positivity
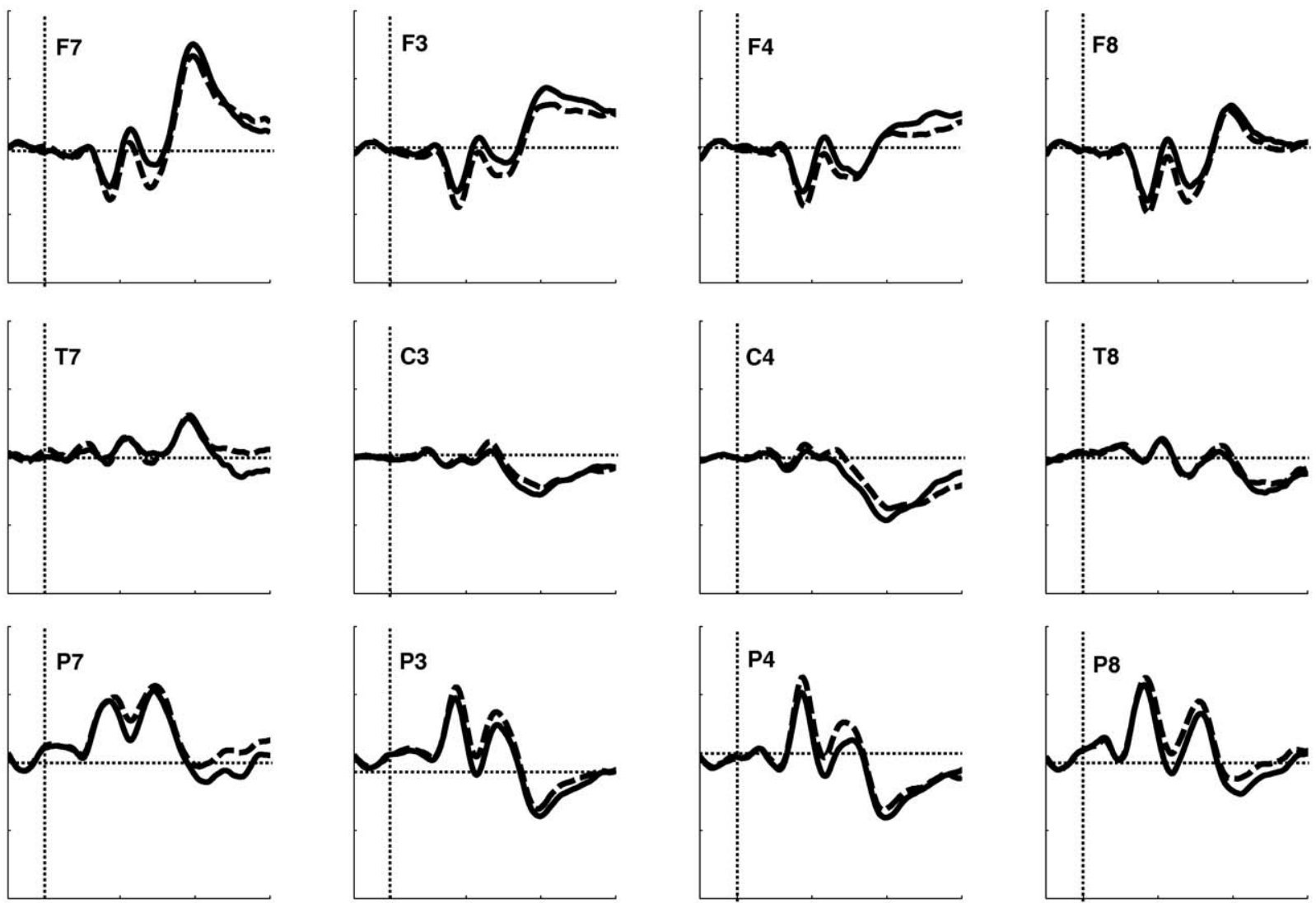

$$
\text { posterior negativity }
$$
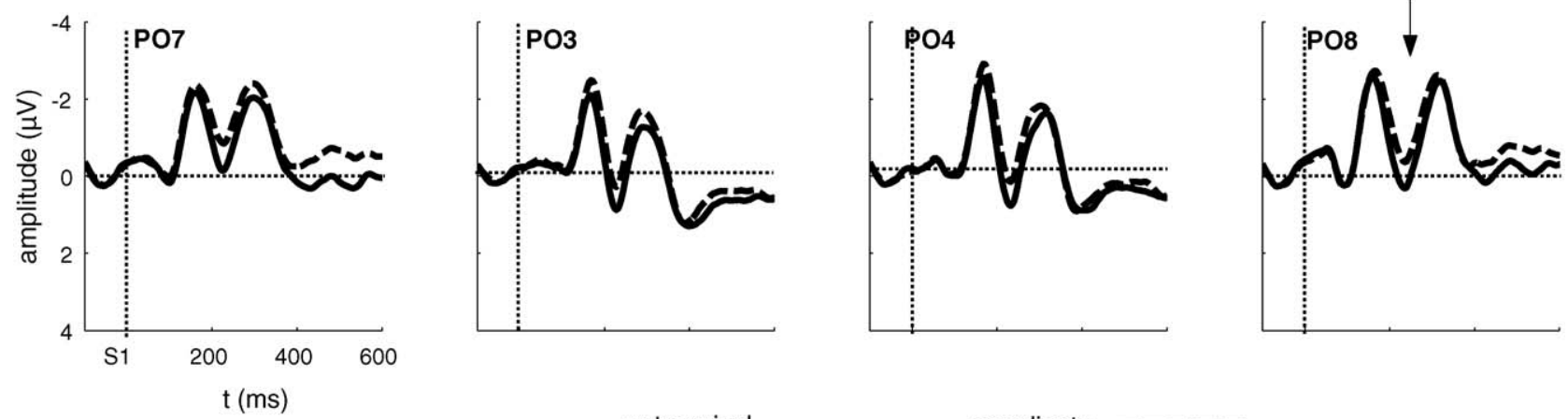

categorical

coordinate -ーーーー

Fig. 3. ERPs elicited by S1 in the categorical and coordinate judgment tasks along the left lateral and medial and right medial and lateral sites (from utmost left panel to utmost right panel), and along the anterior-posterior axis (from upper to lower panel). Task dependent topographical effects are visible at anterior and posterior sites from about 200 to $300 \mathrm{~ms}$ after $\mathrm{S} 1$. 
(A)

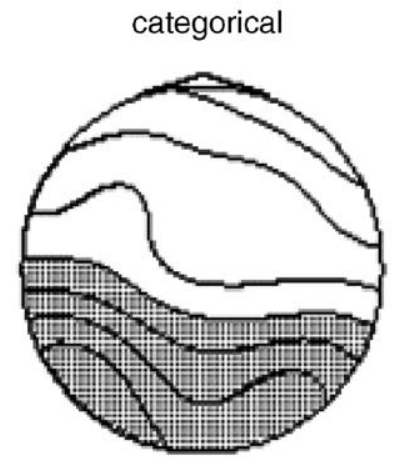

categorical

(B)
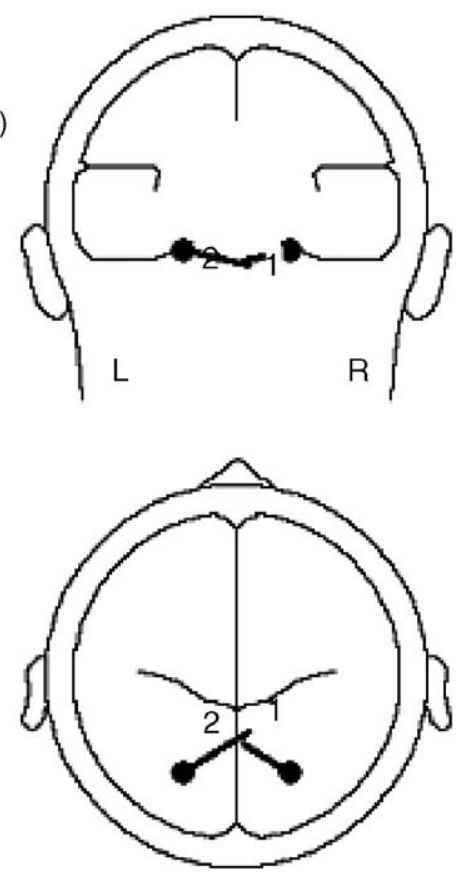

coordinate

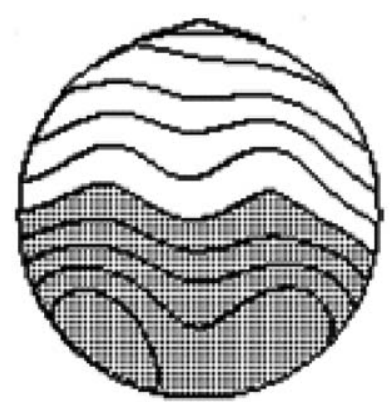

coordinate
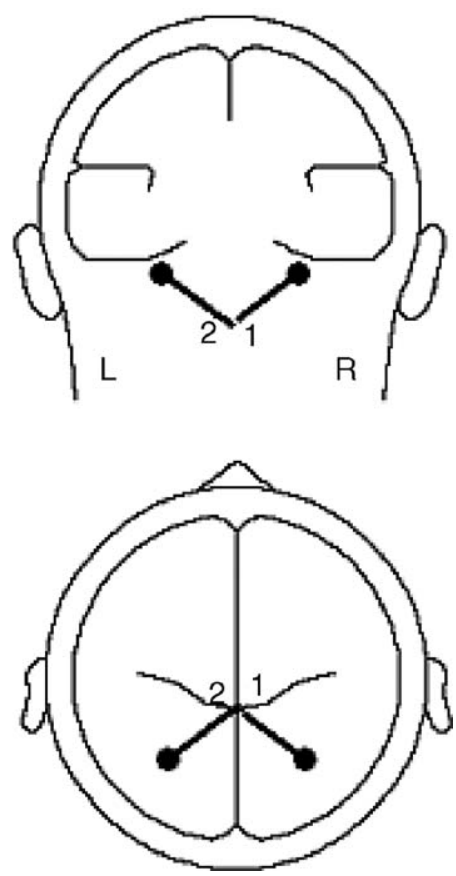

Fig. 4. Spline maps (upper panel A) and estimated dipole sources (lower panel B) for activity at $200 \mathrm{~ms}$ after S1 onset. Spacing for the spline maps amounted to $0.5 \mu \mathrm{V}$ for the categorical and the coordinate conditions (panel A), and negativity is indicated by hatching. The resulting dipole solutions left 4.3 and $1.8 \%$ residual variance in the categorical and coordinate tasks, respectively.

dipole pair (see Fig. 8), of which the contralateral source was indeed more active than the ipsilateral source.

For the question at hand, no significant interactions between electrode and task, and hemisphere and task were obtained, but a significant task $\times$ electrode $\times$ hemisphere interaction was found from 400 to $440 \mathrm{~ms}$. Inspection of Fig. 7 suggests that this interaction may be due to increased positivity (i.e. a $\mathrm{P} 3$ component) above right central sites (i.e. C4) in the categorical condition. Task differences were largest at $404 \mathrm{~ms}$ after S2 onset, both for LVF and for RVF stimuli. The spline maps revealed more positivity in the categorical task within the right-hemisphere than in the coordinate task (see Fig. 9). Source modeling on $404 \mathrm{~ms}$ after S2 onset with one symmetrical dipole pair left less than $4.9 \%$ residual variance. In both tasks, sources were more active on the right than on the left (average dipole moments: left-hemisphere
5.0, right-hemisphere 6.8), but this difference (see Fig. 9) was larger in the categorical task (2.1) than in the coordinate task (1.7).

\section{Discussion}

Coding the spatial relation between two objects may be performed in a precise way (i.e. coordinate) but also at a more abstract level (i.e. categorical). According to the separate spatial coding hypothesis, these two types of spatial coding are qualitatively different, and are performed by the right- and lefthemisphere (Kosslyn et al., 1989), respectively, although later versions indicate that lateralization need not be so strict (Kosslyn et al., 1992). An S1-S2 variant of the Bar Dot task was designed to separate task effects on encoding and memorizing of S1 from 

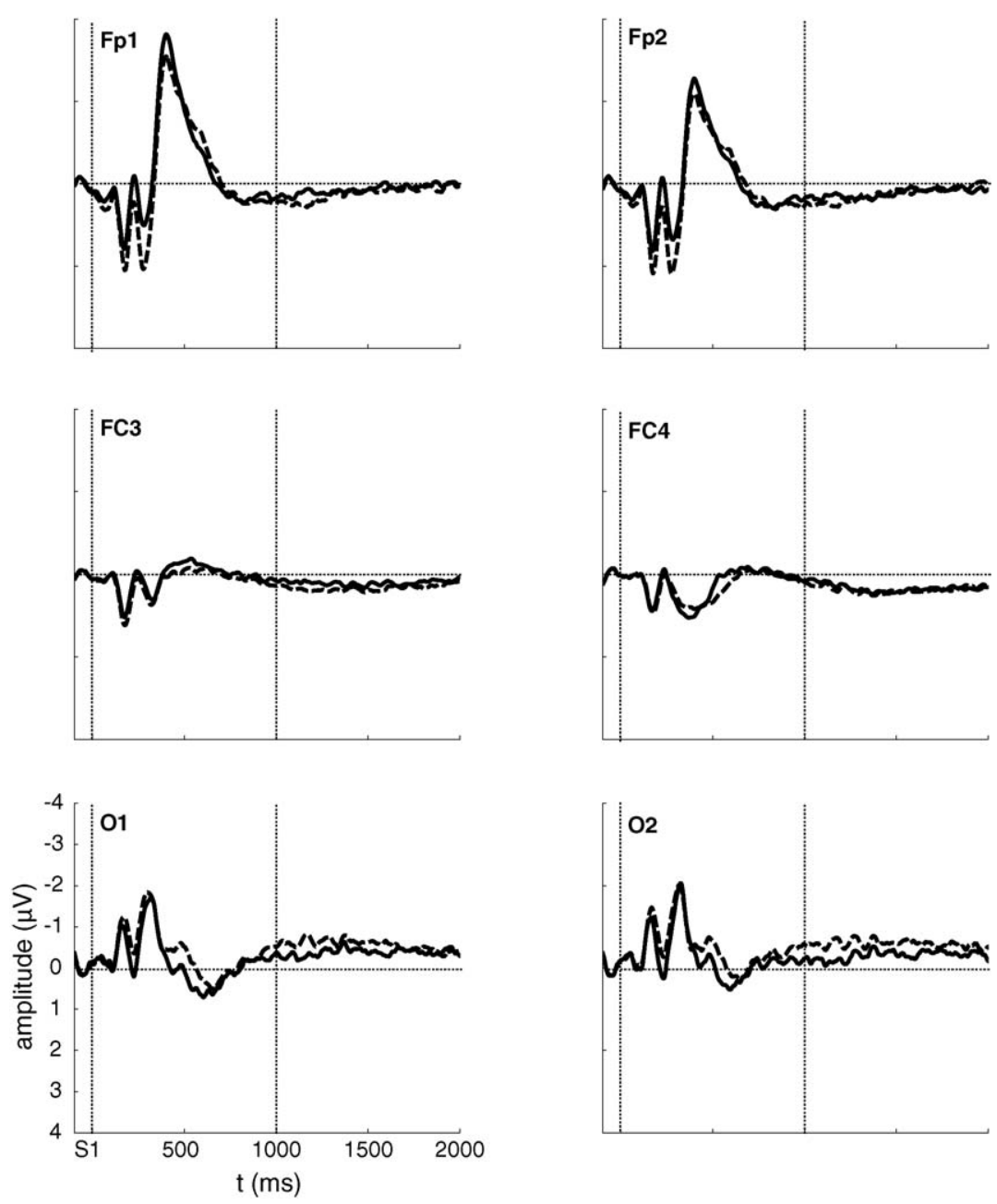

categorical

coordinate ------

Fig. 5. Slow waves in the categorical and coordinate judgment tasks from 1000 to $2000 \mathrm{~ms}$ after S1 for some representative electrodes. Slow anterior positivity and posterior negativity is present from 1000 to $2000 \mathrm{~ms}$ after S1. Increased negativity in the coordinate task seems present at posterior sites.

Table 1

$F$-values for effects including the factors task (T), electrode (E), hemisphere (H), and visual field (VF) for the intervals from 0 to $600 \mathrm{~ms}$ after $\mathrm{S} 2$

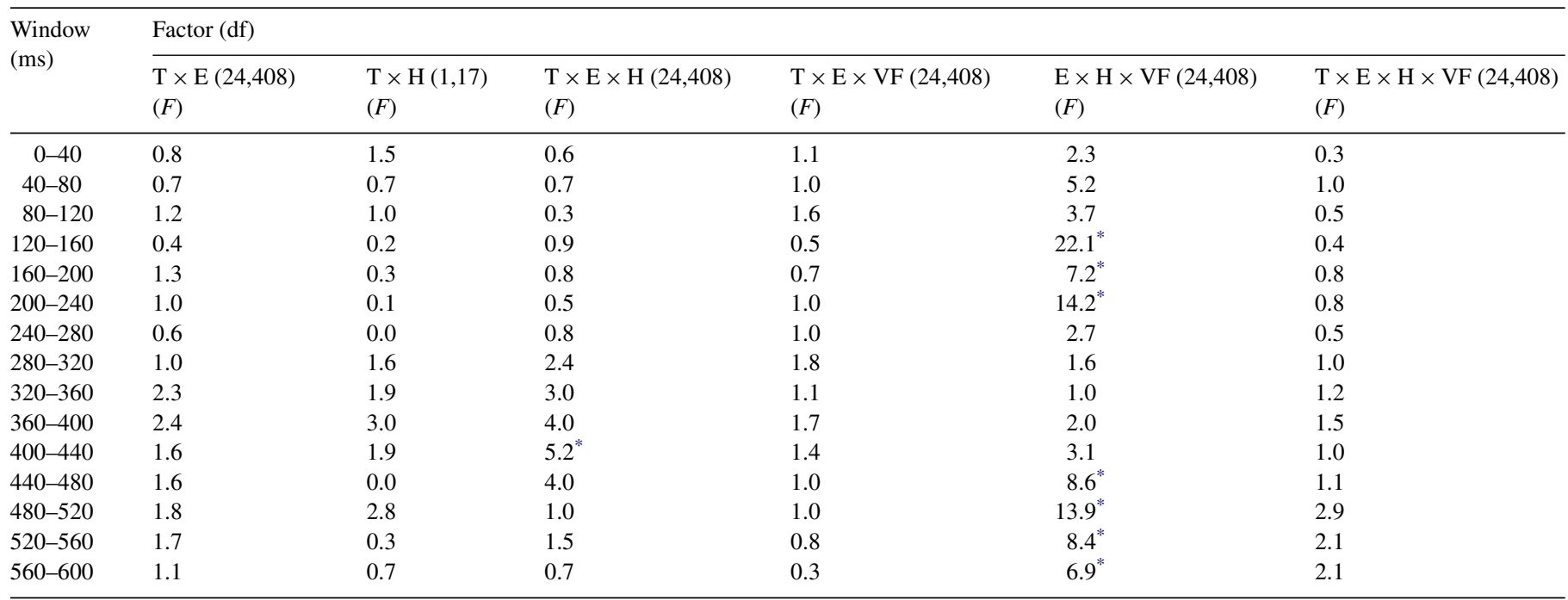

* $p<0.0033$. 
$1064 \mathrm{~ms}$ after S1
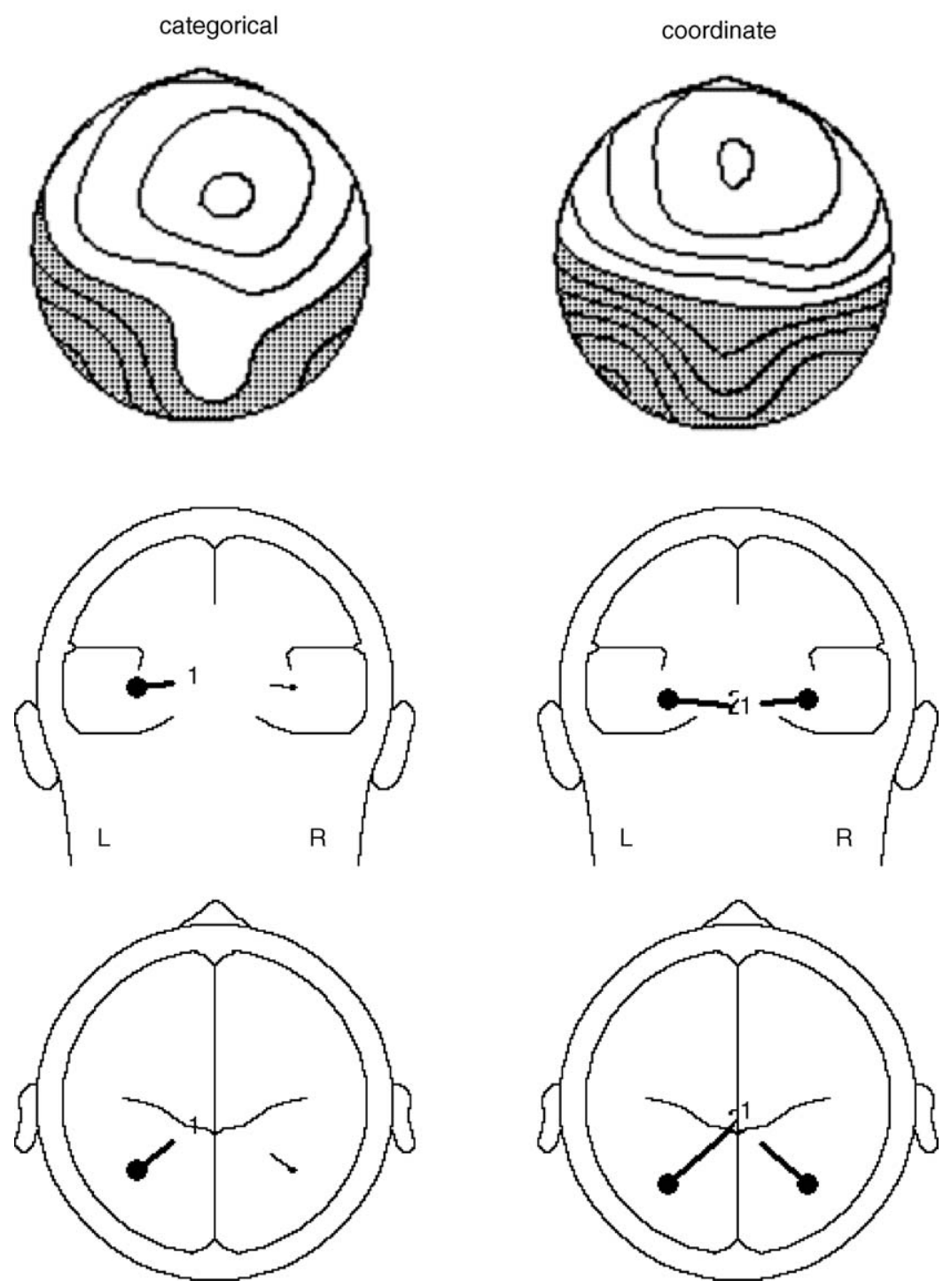

Fig. 6. Spline maps (up) and estimated dipole sources (below) for activity at $1064 \mathrm{~ms}$ after S1 onset. Spacing for the spline maps amounted to $0.2 \mu \mathrm{V}$, and negativity is indicated by hatching. The resulting dipole solutions left 11.8 and $3.7 \%$ residual variance in the categorical and coordinate tasks, respectively.

subsequent retrieval, judgment and motor processes, by looking at the S1-S2 interval in particular, thereby providing more detailed information about the level at which categorical and coordinate processing start to diverge. $\mathrm{S} 2$ was presented in the LVF or RVF which may provide additional behavioral support for the separate spatial coding hypothesis.

The behavioral data (see Fig. 2) revealed faster and more accurate responses for LVF than for RVF stimuli in the coordinate task but no reversal was obtained in the categorical task. As indicated in our introduction, this pattern has been found more often with the Bar Dot task, and provides partial support for the separate spatial coding hypothesis, as it may be considered as indirect evidence for a right-hemispheric specialization for coordinate spatial processing. The additional analyses with the factor block indicated that the observed interaction cannot be ascribed to a differential effect of practice in these tasks. The absence of a reversal of the field effect in the categorical task raises questions on the stability of the visual field effect (see Bruyer et al., 1997), which could be interpreted as a failure of the spatial encoding hypothesis, although one might argue that Bar Dot tasks in general are less well suited to test this part of the hypothesis. Responses were less accurate in the coordinate than in the categorical task. As a consequence, it may be concluded that the coordinate task was more difficult than the categorical task.

ERPs elicited by S1 differed between tasks (see Figs. 3 and 5), which was confirmed by our statistical results, as clear task $\times$ electrode interactions were present from 160 to $320 \mathrm{~ms}$. Specifically, increased anterior positivity and posterior negativity was found in the coordinate as compared to the categorical task. This result resembles the findings of Parrot et al. (1998), and indicates that early task differences can also be observed with centrally presented stimuli within an S1-S2 paradigm. As a consequence, processing of $\mathrm{S} 1$ is indeed affected by the 

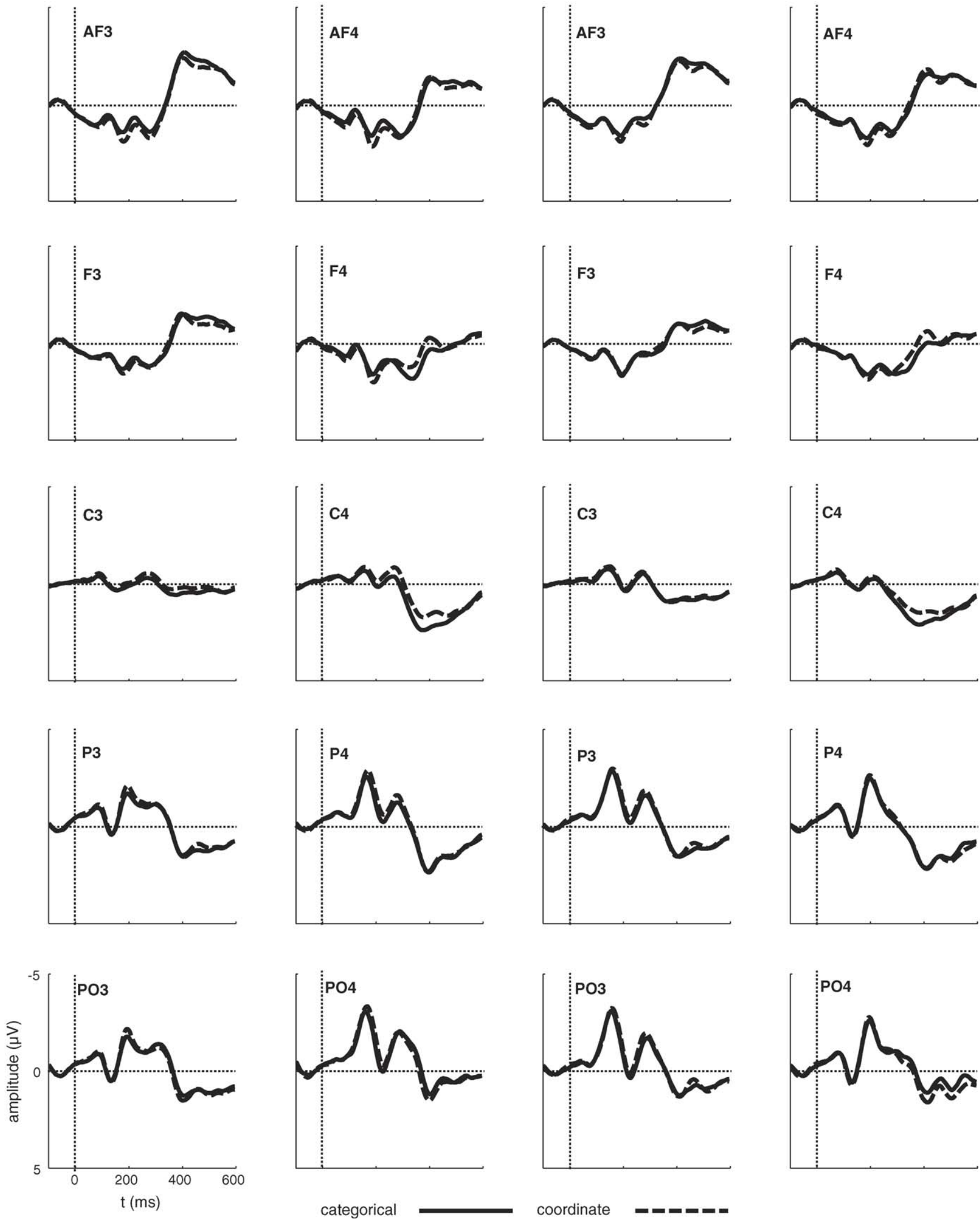

Fig. 7. ERPs in the categorical and the coordinate task elicited by S2 displayed in the left or the right visual field (LVF or RVF, in the left or right panel) for lateral electrodes above the left- and right-hemisphere, along the anterior-posterior axis. Hemispherical differences between tasks seem present at around $400 \mathrm{~ms}$ after $\mathrm{S} 2$ above central sites. 
$168 \mathrm{~ms}$ after S2
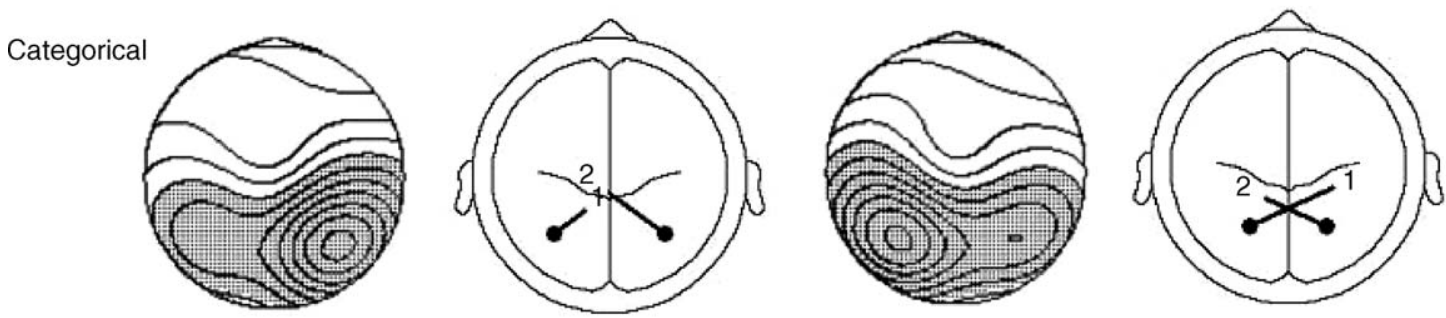

LVF

RVF
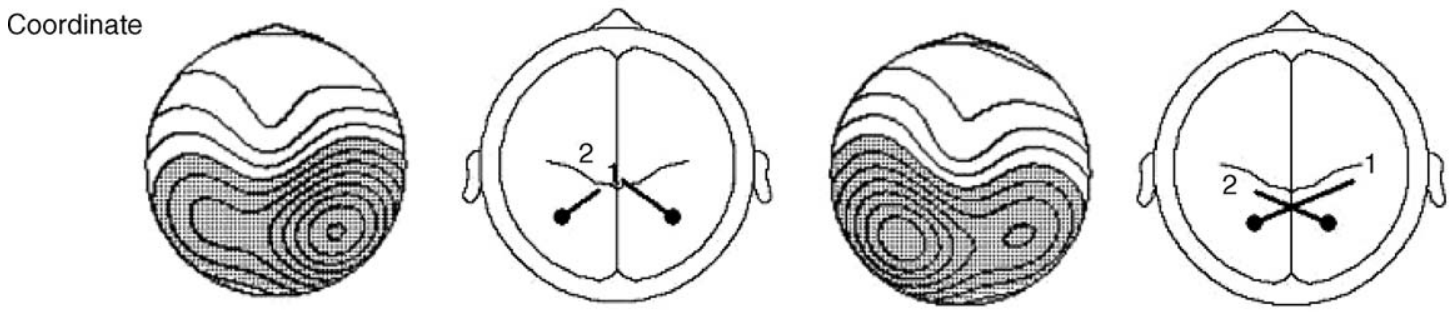

Fig. 8. Results of source analyses with one symmetrical dipole pair for the maximum of the global field power (at $168 \mathrm{ms)}$ ), as a function of task (upper panel vs. lower panel) and visual field of S2 (left or right panel). Residual variance was less than 3.3\%. Spline maps are included (spacing $0.5 \mu \mathrm{V}$ ), which confirm that posterior negativity (hatched) was maximal at the hemisphere contralateral to the visual field in which S2 was displayed.

type of required judgment. Source analyses per task revealed a posterior dipole pair in both tasks, being more active in the coordinate than in the categorical task (see Fig. 4B). Thus, categorical and coordinate spatial processing diverge rather early, which seems to indicate an effect at the level of encoding as it occurred shortly after S1, was largest at posterior sites, and could be explained by a posterior dipole pair. This early divergence seems to have a quantitative rather than a qualitative nature, and is possibly related to the task effect on accuracy indicated above. That is, it may reflect a compensatory strategy, involving extra allocation of attention to processing S1 in the coordinate task, thereby trying to keep performance at an acceptable level in that task.

Analyses on later slow waves within the S1-S2 interval (see Figs. 5 and 6) provided additional information on the involvement of spatial memory in these tasks. We observed task $\times$ electrode interactions from 1000 to $2000 \mathrm{~ms}$ after S1. Source analyses again revealed posterior sources in both tasks, being more active in the coordinate than in the categorical task, which points to the extra involvement of spatial working memory
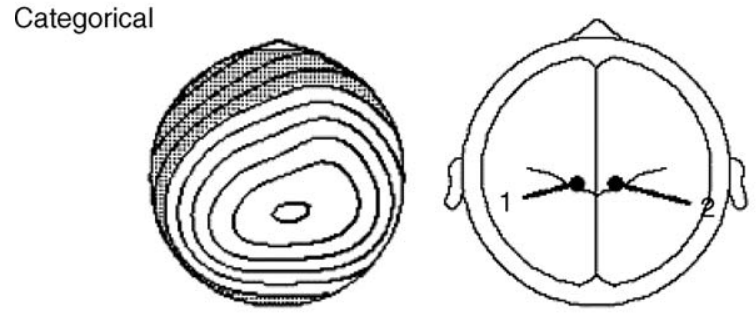

Coordinate

LVF
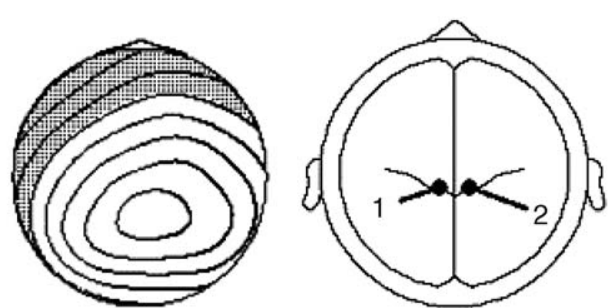
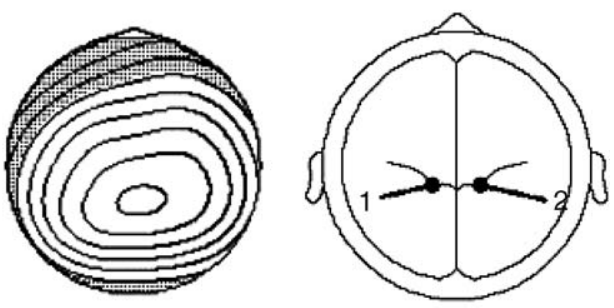

RVF
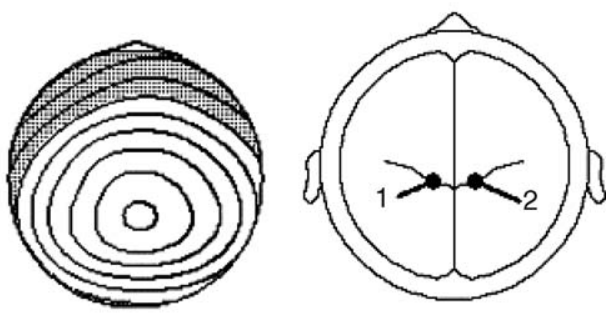

Fig. 9. Spline maps and dipole sources at $404 \mathrm{~ms}$ after S2, as a function of task (upper panel vs. lower panel) and visual field of S2 (left or right panel). Residual variance was less than $5.0 \%$. Spacing for the spline maps amounted to $1.0 \mu \mathrm{V}$. 
in the coordinate task (Bosch et al., 2001). These findings may indicate that with coordinate instructions participants maintain a more precise, detailed spatial image in working memory (possibly related to a compensatory strategy), whereas this tendency may be less under categorical instructions. The slow waves, however, may partially reflect anticipation of the forthcoming fixation point as indexed with the contingent negative variation (CNV; see Brunia, 1999; Verleger, Wauschkuhn, Van der Lubbe, Jaśkowski, \& Trillenberg, 2000). Application of sophisticated paradigms as developed by Bosch et al. (2001) with an intermediate task cue and different memory loads may provide more detailed information on the involvement of spatial working memory in these tasks.

The early ERP effects after S2, being most pronounced at $168 \mathrm{~ms}$ after S2, and the spline maps and results of the source analyses validate the assumption made by the visual half field technique that $\mathrm{S} 2$ initially arrived in the contralateral hemisphere (see Figs. 7 and 8). Task dependent topographical effects were only found from 400 to $440 \mathrm{~ms}$. Inspection of Figs. 7 and 9 suggests that this task effect, being largest at $404 \mathrm{~ms}$ after S2 onset, is due to increased right-hemispheric positivity in the categorical as compared to the coordinate task, which was supported by the results of our source analyses. Thus, a right-hemispheric source seems more active in case of categorical than in case of coordinate processing, which obviously conflicts with the separate spatial coding hypothesis. One might speculate that an additional source with an opposite orientation is involved that is more active in the coordinate task, which may explain the reduced positivity. However, given the small amount of variance that remained after source analyses with one dipole pair, this possibility seems less favorable. Alternatively, one could argue that the process reflects inhibition of right-hemispheric coordinate processing during categorical spatial processing, which in some way would correspond with the separate spatial coding hypothesis. Nevertheless, this explanation seems a bit far-fetched. Given the fact that no task dependent effect with a comparable latency was found after S1, that it was independent from the visual field in which S2 appeared, and that it occurred rather late, it may be proposed that the process involved was either related to retrieval of S1 from spatial memory, or from succeeding judgment and motor processes, but probably not to encoding of S2.

Based on the previous discussion, it appears that our support for the separate spatial coding hypothesis is rather scarce. While a visual field effect on our behavioral measures was evidently present in the coordinate task, and S2 initially arrived in the contralateral hemisphere, no electrophysiological support was found that the right-hemisphere is more active in the coordinate task. One could argue that this failure is due to the lower spatial resolution of EEG (although this is still in the range of a few $\mathrm{cm}$, and we could show that $\mathrm{S} 2$ initially arrived in the contralateral hemisphere), however, the reported fMRI and PET evidence may reflect effects that are less well highlighted in our study. For example, it may be conjectured that the observed effects in fMRI and PET studies reflect tonic changes in activity, which have no influence on phasic stimulus-induced effects highlighted by our study. A way to examine this is to vary task instructions on a trial-by-trial basis (see Bosch et al., 2001) rather than by using separate tasks as in the current study, and to compare activity induced by categorical and coordinate task instructions.

Closer inspection of the fMRI results of Baciu et al. (1999), who, like in our study, presented a bar and dot in the center, reveals that right-hemispheric activation in the coordinate task reversed to increased left-hemispheric activation over time, which they attributed to practice and the building up of new categorical representations. However, they acknowledged that the effect in the left-hemisphere might also reflect an ability of the left-hemisphere to perform coordinate computations, so their evidence seems not so decisive.

The point remains that the visual field effect requires an explanation: what is the source of more accurate responses in the coordinate task for stimuli presented in the LVF? Instead of ascribing this visual field effect to the aforementioned tonic changes depending on the task at hand, it may be argued that the effect is related to hardwired processing differences between the two hemispheres. For example, Kosslyn et al. (1998) stated in their PET study that "... .our failure to find the lateralized parietal differences we expected may indicate that the relevant processing is fast and "automatic" ...". Indeed, visual neurons in the left-hemisphere are thought to have small receptive fields, whereas visual neurons in the right-hemisphere are thought to have large and overlapping receptive fields, and due to these differences encoding the precise location of an object relative to another object may be more accurate when stimuli fall in the LVF than when they fall in the RVF (for details see Chabris \& Kosslyn, 1998; Jager \& Postma, 2003). Furthermore, due to these differences in spatial resolution, coordinate judgments may simply be more accurate and easier when stimuli fall within the LVF, whereas this aspect seems less relevant when categorical judgments are required.

Regarding our categorical task, no support was found for a visual field effect on our behavioral measures. Although several other studies were also unable to demonstrate the effect (see Section 1), one might argue that this is because of our slight modification of the original paradigm. Presentation of to be judged stimuli in the center does not seem problematic, given the findings of Baciu et al. (1999), as they found left-hemispheric activation in their categorical task. Another change concerns the use of an S1-S2 paradigm, but, although this manipulation is new for the Bar Dot task, S1-S2 paradigms were rather effective in demonstrating the visual field effect in case of categorical tasks (Laeng, 1994; Laeng \& Peters, 1995). As a consequence, we do not believe that our failure to obtain a visual field effect in the categorical task is due to our slight modification of the task.

In conclusion, by employing an S1-S2 paradigm, we had the ability to separate spatial task effects on encoding and memory processes from subsequent judgment and motoric processes. Support was found for the idea that categorical and coordinate processing diverge both while encoding and memorizing the spatial relation between two objects. This divergence seems to have a quantitative rather than a qualitative nature, and may be due to a compensatory strategy because of increased task difficulty in the coordinate task. LVF and RVF stimuli were shown to be initially processed in the contralateral hemisphere, and a visual field effect was found in the coordinate task, which may 
be related to hardwired processing differences between the leftand right-hemisphere, rather than differential activation of the two hemispheres due to different task demands.

\section{Acknowledgements}

The current study was supported by a grant from The Netherlands Organization for Fundamental Research (NWO: 440-20000). A first analysis based on the data of twelve participants was presented by Marieke Schölvinck at the 2nd Dutch EndoNeuroPsycho meeting in Doorwerth, the Netherlands (June 2003). Thanks are due to Bruno Laeng, two anonymous reviewers, Matthijs Noordzij for comments on an earlier version of the present paper, and to Daan Vroon for his help in measuring additional participants.

\section{References}

Annett, M. (1970). A classification of hand preference by association analysis. British Journal of Psychology, 61, 303-321.

Awh, E., Jonides, J., \& Reuter-Lorenz, P. A. (1998). Rehearsal in spatial working memory. Journal of Experimental Psychology: Human Perception and Performance, 24, 780-790.

Baciu, M., Koenig, O., Vernier, M. P., Bedoin, N., Rubin, C., \& Segenbarth, C. (1999). Categorical and coordinate spatial relationships: fMRI evidence for hemispheric specialization. NeuroReport, 10, 1-6.

Banich, M. T., \& Federmeier, K. D. (1999). Categorical and metric spatial processes distinguished by task demands and practice. Journal of Cognitive Neuroscience, 11, 153-166.

Biederman, I. (1987). Recognition-by-components: A theory of human image understanding. Psychological Review, 94, 115-147.

Bosch, V., Mecklinger, A., \& Friederici, A. D. (2001). Slow cortical potentials during retention of object, spatial, and verbal information. Cognitive Brain Research, 10, 219-237.

Briggs, G. G., \& Nebes, R. D. (1975). Patterns of hand preference in a student population. Cortex, 11, 230-238.

Brunia, C. H. M. (1999). Neural aspects of anticipatory behavior. Acta Psychologica, 101, 213-242.

Bruyer, R., Scailquin, J. C., \& Coibion, P. (1997). Dissociation between categorical and coordinate spatial computations: Modulation by cerebral hemispheres, task properties, mode of response, and age. Brain and Cognition, 33, 245-277.

Chabris, C. F., \& Kosslyn, S. M. (1998). How do the cerebral hemispheres contribute to encoding spatial relations. Current directions in Psychological Science, 7, 8-14.

Cowin, E. L., Roth, E., \& Hellige, J. B. (1994). Spatial processing and hemispheric asymmetry: Contributions of the transient/magnocellular visual system. Journal of Cognitive Neuroscience, 10, 472-483.

Gratton, G., Coles, M. G. H., \& Donchin, E. (1983). A new method for off-line removal of ocular artifacts. Electroencephalography and Clinical Neurophysiology, 55, 468-484.

Hellige, J. B., \& Michimata, C. (1989). Categorization versus distance: Hemispheric differences for processing spatial information. Memory and Cognition, 17, 770-776.

Hellige, J. B., \& Cumberland, N. (2001). Categorical and coordinate spatial processing: More on contributions of the transient/magnocellular visual system. Brain and Cognition, 45, 155-163.

Jager, G., \& Postma, A. (2003). On the hemispheric specialization for categorical and coordinate spatial relations: A review of the current evidence. Neuropsychologia, 41, 504-515.

Jha, A. P. (2002). Tracking the time-course of attentional involvement in spatial working memory: An event-related potential investigation. Cognitive Brain Research, 15, 61-69.
Kemmerer, D., \& Tranel, D. (2000). A double dissociation between linguistic and perceptual representations of spatial relationships. Cognitive Neuropsychology, 17, 393-414.

Kosslyn, S. M. (1987). Seeing and imagining in the cerebral hemispheres: A computational approach. Psychological Review, 94, 148-175.

Kosslyn, S. M., Koenig, O., Barrett, A., Cave, C., Tang, J., \& Gabrieli, J. D. E. (1989). Evidence for two types of spatial representations: Hemispheric specialization for categorical and coordinate relations. Journal of Experimental Psychology: Human Perception and Performance, 15, 723-735.

Kosslyn, S. M., Chabris, C. F., Marsolek, C. J., \& Koenig, O. (1992). Categorical versus coordinate spatial relations: Computational analyses and computer simulations. Journal of Experimental Psychology: Human Perception and Performance, 18, 562-577.

Kosslyn, S. M., Thompson, W. I., Gitelman, D. R., \& Alpert, N. M. (1998). Neural systems that encode categorical versus coordinate spatial relations: PET investigations. Psychobiology, 26, 333-347.

Laeng, B. (1994). Lateralization of categorical and coordinate spatial functions: A study of unilateral stroke patients. Journal of Cognitive Neuroscience, 6, 189-203.

Laeng, B., Shah, J., \& Kosslyn, S. M. (1999). Identifying objects in conventional and contorted poses: Contribution of hemisphere-specific mechanisms. Cognition, 70, 53-85.

Laeng, B., \& Peters, M. (1995). Cerebral lateralization for the processing of spatial coordinates and categories in left- and right-handers. Neuropsychologia, 33, 421-439.

Mangun, G. R., Luck, S. J., Plager, R., Loftus, W., Hillyard, S. A., Handy, T., et al. (1994). Monitoring the visual world-hemispheric asymmetries and subcortical processes in attention. Journal of Cognitive Neuroscience, 6, 267-275

Michimata, C. (1997). Hemispheric processing of categorical and coordinate spatial relations in vision and visual imagery. Brain and Cognition, 24, 539-556.

Parrot, M., Doyon, B., \& Cardebat, D. (1998). Hemispheric specialization for coordinate versus categorical spatial processing: An ERP study. Neurolmage, $7, \mathrm{~S} 332$.

Parrot, M., Doyon, B., Demonet, J. F., \& Cardebat, D. (1999). Hemispheric preponderance in categorical and coordinate visual processes. Neuropsychologia, 37, 1215-1225.

Postma, A., Izendoorn, R., \& De Haan, E. H. F. (1998). Sex differences in object location memory. Brain and Cognition, 36, 334-345.

Ruchkin, D. S., Johnson, J. R., Grafman, J., Canoune, H., \& Ritter, W. (1997). Multiple visuospatial working memory buffers: Evidence from spatiotemporal patterns of brain activity. Neuropsychologia, 35, 195-209.

Ruchkin, D. S., Johnson, R., Grafman, H., Canoune, W., \& Ritter, W. (1992). Distinctions and similarities among working memory processes: An eventrelated potential study. Cognitive Brain Research, 1, 53-66.

Rybash, J. M., \& Hoyer, W. J. (1992). Hemispheric specialization for categorical and coordinate spatial representations: A reappraisal. Memory and Cognition, 20, 271-276.

Scherg, M. (1990). Fundamentals of dipole source potential analysis. In F. Grandori, M. Hoke, \& G. L. Romani (Eds.), Auditory evoked magnetic fields and potentials (pp. 40-69). Basel: Karger.

Sergent, J. (1991a). Judgments of relative position and distance on representations of spatial relations. Journal of Experimental Psychology: Human Perception and Performance, 91, 762-780.

Sergent, J. (1991b). Processing of spatial relations within and between the disconnected cerebral hemispheres. Brain, 114, 1025-1043.

Trojano, L., Grossi, D., Linden, D. E. J., Formisano, E., Goebel, R., Cirillo, S., et al. (2002). Coordinate and categorical judgments in spatial imagery. An fMRI study. Neuropsychologia, 40, 1666-1674.

Verleger, R., Wauschkuhn, B., Van der Lubbe, R. H. J., Jaśkowski, P., \& Trillenberg, P. (2000). Posterior and anterior contribution of hand-movement preparation to late CNV. Journal of Psychophysiology, 14, 69-86.

Wilkinson, D., \& Donnelly, N. (1999). The role of stimulus factors in making categorical and coordinate spatial judgments. Brain and Cognition, 39, 171-185. 